\title{
Ovarian preservation and prognosis in adnexal torsion surgery - a retrospective analysis
}

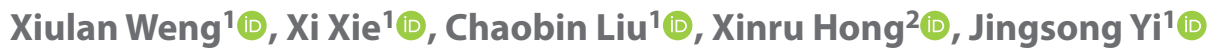 \\ ${ }^{1}$ Department of Gynaecology, Fujian Maternity and Child Health Hospital, \\ Affiliated Hospital of Fujian Medical University, Fuzhou, China \\ ${ }^{2}$ Department of Obstetrics and Gynaecology, 900 Hospital of the Joint Logistics Team, Fuzhou, China
}

\begin{abstract}
Objectives: This study aims to analyze the conditions of ovarian preservation during adnexal torsion surgery, and safety of ovarian preservation.

Material and methods: A retrospective analysis of 130 patients, who underwent surgery for ovarian benign tumor pedicle torsion in Fujian Provincial Maternal and Child Health Hospital from June 2013 to June 2018, was conducted. This study analyses the possible risk factors affecting the operation method using multiple logistic regression and analyses the complications and the recovery of ovarian function after the treatment of the ovarian preservation.

Results: Among these patients, 58 received ovarian cystectomy, while 72 received ovariectomy. There was no significant difference in terms of age, preoperative blood, operation time and surgical bleeding volume between the two groups $(p>0.05)$. However, there was a significant difference in preoperative adnexal blood flow, abdominal pain to the surgical interval, and a collection of torsion cycles $(p<0.05)$. There was an increased risk of ovarian resection in patients whose blood flow of the annex disappeared, whose time of abdominal pain was long, and whose number of twists were significant. For the preservation group, there were no increases in postoperative complications.

Conclusions: According to clinical indicators, such as preoperative adnexal blood flow, abdominal pain to the interval of surgery and the number of torsion cycles, it was determined whether it was feasible to keep the ovary. Retaining the ovary is safe, effective and feasible in adnexal torsion.
\end{abstract}

Key words: appendix torsion; ovarian preservation; postoperative follow-up; surgical management

Ginekologia Polska 2020; 91, 5: 277-280

\section{INTRODUCTION}

The torsion of female fallopian tubes, ovaries, or both is called adnexal torsion (AT), which account for $2.7 \%$ of all gynecologic acute abdomen cases [1], while $71 \%$ of cases occur in women of childbearing age [2]. In traditional surgery, the affected adnexa should be resected to prevent the risk of acute pulmonary embolism. The removal of adnexal masses may effect female fertility and endocrine function [3]. With the development of medicine, conservative surgery for maintaining ovarian preservation in young women has been given increasing attention in recent years, but it remains difficult to solve the problem on how to avoid thromboembolism and ensure the recovery of ovarian blood supply function [4]. The present study retrospectively classifies 130 cases of adnexal torsion cases in Fujian Provincial Maternal and
Child Health Hospital from June 2013 to June 2018. Furthermore, the possible conditions for ovarian preservation and the follow-up records of the prognosis of patients were analyzed and summarized.

\section{MATERIAL AND METHODS}

\subsection{General information}

From June 2013 to June 2018, Fujian Maternal and Child Health Hospital admitted 130 cases of adnexal torsion, who were within the 12-45 years old age range (after menarche-before menopause). These patients underwent routine preoperative examination to eliminate surgical contraindications. Furthermore, adequate information was collected from all patients prior to the surgery. Patients who needed ovarian preservation are willing to bear the risk 
Table 1. Comparison of the general information for the preservation group and resection group (mean \pm SD)

\begin{tabular}{|l|l|l|l|l|l|}
\hline Group & Cases & Age [y] & Mean diameter of tumor $[\mathbf{c m}]$ & Duration of Torsion [h] & Intraoperative blood loss [mL] \\
\hline Preservation & 58 & $27.05 \pm 7.37$ & $7.475 \pm 2.40$ & $31.40 \pm 24.03$ & $60.00 \pm 35.06$ \\
\hline Resection & 72 & $30.74 \pm 8.64$ & $8.384 \pm 2.58$ & $42.65 \pm 41.10$ & $51.20 \pm 40.10$ \\
\hline t & & -2.227 & -1.816 & -1.100 & 0.615 \\
\hline p & & 0.068 & 0.894 & 0.288 & 0.195 \\
\hline
\end{tabular}

Table 2. Analysis of other factors in the preservation group and resection group

\begin{tabular}{|l|l|l|l|l|l|}
\hline Factors & $\boldsymbol{\beta}$ & $\mathbf{P}$ & $\mathbf{O R}$ & $\mathbf{9 5 \%} \mathbf{C l}$ \\
\hline Preoperative blood picture & 0.156 & 0.764 & 1.169 & 0.421 & 3.244 \\
\hline Operation time & -0.110 & 0.754 & 0.896 & 0.451 & 1.782 \\
\hline Interval from abdominal pain to operation & -0.016 & 0.017 & 0.984 & 0.971 & 0.997 \\
\hline Intraoperative blood loss & 0.001 & 0.821 & 1.001 & 0.994 & 1.008 \\
\hline Twists number & -0.576 & 0.037 & 0.562 & 0.372 & 0.967 \\
\hline Attachment blood flow & -1.160 & 0.000 & 0.313 & 0.181 & 0.542 \\
\hline
\end{tabular}

$\mathrm{B}$ - Regression coefficients; $\mathrm{p}$ - Significance ( $\mathrm{p}$-value); $\mathrm{OR}$ - Odds ratio; $\mathrm{Cl}$ - confidence interval

Table 3. Comparison of general conditions after the operation between the two groups (mean \pm SD)

\begin{tabular}{|l|l|l|l|l|}
\hline Group & Cases & WBC $(\wedge 109 / L)$ & $\operatorname{Max} \mathbf{T}\left[{ }^{\circ} \mathbf{C}\right]$ & $\begin{array}{l}\text { Days in } \\
\text { hospital }\end{array}$ \\
\hline Preservation & 58 & $10.09 \pm 3.46$ & $37.29 \pm 0.42$ & $5.9 \pm 2.01$ \\
\hline Resection & 72 & $9.68 \pm 3.09$ & $37.30 \pm 0.34$ & $8.7 \pm 2.07$ \\
\hline $\mathrm{t}$ & & 0.637 & -0.159 & -2.525 \\
\hline $\mathrm{p}$ & & 0.921 & 0.094 & 0.029 \\
\hline
\end{tabular}

WBC — white blood cell count; Max T — Postoperative maximum body temperature

of postoperative thromboembolic disease, ovarian re-distortion, necrosis, infection and secondary surgery. Among these patients, 58 received ovarian cystectomy (preservation group) and 72 received adnexal resection (resection group). Table 1 presents the overall situation of the two groups.

\subsubsection{General treatment}

Patients in both groups, who provided adequate information prior to surgery, received laparotomy or laparoscopic surgery. All pathological findings were benign. Each patient received anti-inflammatory symptomatic treatment and subcutaneous injection of low molecular weight heparin of 2,500-5,000 units daily, until discharge.

\subsubsection{Follow-up}

Postoperative follow-ups were conducted to determine whether there are any complications in patients with ovarian preservation, such as thrombosis, concurrent infection and secondary surgery. In order to evaluate the recovery of ovarian function, these patients were followed up for two years after surgery for menstruation, restoration of blood supply, menstruation and dominant follicles that affect the adnexa, and basic endocrine. The fertility status of women with fertility needs were also determined.

\subsubsection{Statistical methods}

SPSS 23.0 software was used for the statistical analysis. Data were analyzed by t-test, unconditional single factor, and multi-factor logistic regression. $\mathrm{P}<0.05$ was considered statistically significant.

\section{RESULTS}

1. There were no statistically significant differences in terms of age at onset, time of operation, blood loss during the operation and tumor size between the two groups (Tab. 1). However, there were statistically significant differences in the number of torsion cycles, the interval from onset to the operation, and in the adnexal blood flow signals before the operation (Tab. 2).

2. There were no statistically significant differences in blood picture and maximum temperature between the resection group and preservation group, while the difference in time of postoperative hospitalization was statistically significant (Tab. 3).

3. Laparoscopic surgery was performed for 39 patients (39/58) in preservation group and 26 patients (26/72) in the resection group. In the preservation group, there were no serious complications, such as septicemia thromboembolic events and infarction. At one month after the operation, the color doppler ultrasonography examination indicated that the blood flow of the affected side was restored. After 2-3 months, 
the average levels of basic hormones were as follows: E2 of $30.21 \pm 5.78 \mathrm{pg} / \mathrm{mL}, \mathrm{FSH}$ of $4.68 \pm 0.66 \mathrm{IU} / \mathrm{L}$, LH of $5.01 \pm 0.98 \mathrm{IU} / \mathrm{L}$. Furthermore, the menstruation recovered within three months, and dominant follicles were observed in 2-6 months by color Doppler ultrasonography. Among these cases, 33 had a successful pregnancy and the delivery was within two years after the operation. Furthermore, no abortion or premature delivery occurred.

\section{DISCUSSION}

Pedicle torsion of ovarian cyst often takes ovarian ischemia as the main pathophysiological change, accompanied by necrosis and infection [5]. Since the torsion of the ovarian cyst pedicle has a higher risk of rupture, embolism and even death, it is clinically suggested that the operation should be performed as soon as possible after the diagnosis, and that the adnexectomy should be performed after clamping the pedicle of the tumor [6]. With the aggravation of the tendency of younger onset of ovarian cysts in recent years, the ovarian preservation in the pedicle torsion of ovarian cysts has gained increasing attention, while the conditions for ovarian preservation have been rarely discussed [7]. In the present study, it was found that the number of torsion cycles, preoperative blood flow of the adnexa, and the interval from onset time to operation were lesser, better and shorter in the preservation group, when compared to the resection group. Studies have shown that when the onset time is short, the number of torsion cycles is few. Furthermore, the preoperative color Doppler ultrasonography indicated that there were blood flow signals in the adnexa, and that young patients, excluding malignant risk, could receive adnexal preservation operation, while postoperative complications should be closely followed up. Once torsion of the adnexal cyst is diagnosed, emergency surgery is required in principle. Meanwhile, in order to prevent the thrombus from falling off and leading to thromboembolism, the operator usually adopts the resection of the affected adnexa as a traditional method [6]. During the operation, the pedicle of the torsion is clamped and removed, which is not feasible for torsion reduction [8]. However, in recent years, with the increasing awareness of the protection of ovarian function, the torsion of the adnexa has been increasingly preserved during the operation. Recent reports have indicated that thromboembolism complications (such as pulmonary embolism) does not increase after the reduction of adnexal torsion $[9,10]$. In fact, the incidence of pulmonary embolism in the case of adnexal torsion is very low (some studies report an incidence of $0.2 \%$ ) [11], and thromboembolic events after conservative surgery only rarely occur. In the present study, the preservation surgery of the adnexa was performed on 58 patients, and the reduction of the torsion followed by cyst removal was performed during the operation. There were no serious complications caused by the thrombus detachment. Furthermore, there were no significant signs of infection in any of the cases after the operation, and there were no statistically significant differences in the highest postoperative temperature and white blood cell count between the preservation group and resection group. However, the length of stay was significantly lower in the preservation group than in the resection group, and the difference was statistically significant. Therefore, it can be considered that the reduction of torsion does not necessarily lead to complications, such as thromboembolism and serious infection.

With the deepening of the understanding of this disease, more and more scholars have tended to perform ovarian cyst resection and reduction. Some scholars have considered that the removal of the ovarian reduction cyst can be performed after the arteriovenous ligation of the affected side. On the one hand, this can prevent the venous thrombosis from falling off and causing an embolism [12]. On the other hand, this preserves the patient's adnexa. However, this destroys the main blood vessels of the ovaries, and has a significant impact on the ovarian function of the affected side. In the present study, the surgery in the preservation group was successfully performed, and the blood vessels were not damaged. Furthermore, the postoperative basal endocrine hormone level did not exhibit a decline in ovarian function. Those who had fertility needs, excluding other factors that may cause infertility, had a successful pregnancy within two years after the operation. These results show that conservative surgery of the adnexa torsion can better protect the fertility and endocrine function of patients.

In the past, the laparotomy exploration was a standard surgical procedure for adnexal torsion. In recent years, with the development of laparoscopy, and the advantages of rapid postoperative recovery, most scholars have supported laparoscopic surgery to rescue the torsion of the ovary [13]. In the present study, laparoscopic surgery was performed on 39 patients (39/58) in the preservation group and on 26 patients $(26 / 72)$ in the resection group, and the average length of hospital stay was significantly shorter, when compared to the laparotomy. The difference was statistically significant ( $5.03 \pm 2.62$ vs $6.82 \pm 3.33, p=0.020)$. Therefore, the preferred surgical procedure for ovarian benign tumor pedicle torsion is laparoscopic exploration [14].

In summary, although surgeries, such as the torsion reduction of adnexa cysts, cannot be considered completely safe, as far as the existing clinical studies are concerned, it can be considered that this type of surgery is of low risk and controllable. The number of torsion cycles, the onset time and the signal of preoperative blood flow are the key reference factors that allowed the investigators to make these clinical decisions. For patients with fertility desires, the func- 
tion of the affected adnexa should be preserved as much as possible. However, the article also has its limitations. For example, retrospective studies cannot analyze causality and the sample size is limited. So more strictly designed, rigorously controlled, and large-scale multi-center clinical trials are still needed to determine how to effectively distinguish reversible ischemic adnexa from irreversible necrotic adnexa and identify laboratory indicators that can objectively reflect the vitality of adnexal torsion with good applicability.

\section{Acknowledgements}

The authors are grateful to Wenchun Xue, Libo Xue and Fengning Lin of the Medical Records Room of Fujian Provincial Maternal and Child Health Hospital, and Lihua Chen of Fujian Medical University.

\section{Conflict of interest}

The authors declare no conflict of interest.

\section{REFERENCES}

1. Spinelli C, Piscioneri J, Strambi S. Adnexal torsion in adolescents: update and review of the literature[J]. Curr Opin Obstet Gynecol. 2015; 27(5): 320-325.

2. Fujishita A, Araki H, Yoshida S, et al. Outcome of conservative laparoscopic surgery for adnexal torsion through one-stage or two-stage operation. Journal of Obstetrics and Gynaecology Research. 2014; 41(3): 411-417, doi: 10.1111/jog.12534.

3. Moribata Y, Kido A, Yamaoka T, et al. MR imaging findings of ovarian torsion correlate with pathological hemorrhagic infarction. Journal of Obstetrics and Gynaecology Research. 2015; 41(9): 1433-1439, doi: 10.1111/jog.12717.

4. Yan X, Xianling Z, Ting Y. Comparison of clinical features of ovarian cyst torsion in postmenopausal and childbearing age patients. Chinese Journal of Women and Children Health Research. 2016; 27(11): 1399-1401.

5. Childress K, Dietrich J. Pediatric Ovarian Torsion. Surgical Clinics of North America. 2017; 97(1): 209-221, doi: 10.1016/j.suc.2016.08.008.

6. Ashwal E, Krissi H, Hiersch L, et al. Presentation, Diagnosis, and Treatment of Ovarian Torsion in Premenarchal Girls. Journal of Pediatric and Adolescent Gynecology. 2015; 28(6): 526-529, doi: 10.1016/j.jpag.2015.03.010.

7. Rastogi D, Yadav A, Hariprasad S, et al. Neonatal ovarian cyst with torsion - A case report. Current Medicine Research and Practice. 2015; 5(1): 26-28, doi: 10.1016/j.cmrp.2015.02.001.

8. Santos X, Cass D, Dietrich J. Outcome Following Detorsion of Torsed Adnexa in Children. Journal of Pediatric and Adolescent Gynecology. 2015; 28(3): 136-138, doi: 10.1016/j.jpag.2014.04.002.

9. Chu K, Zhang Q, Sun N, et al. Conservative laparoscopic management of adnexal torsion based on a 17-year follow-up experience. Journal of International Medical Research. 2018; 46(4): 1685-1689, doi: 10.1177/0300060517754025.

10. Spinelli C, Buti I, Pucci V, et al. Adnexal torsion in children and adolescents: new trends to conservative surgical approach - Our experience and review of literature. Gynecological Endocrinology. 2012; 29(1): 54-58, doi: 10.3109/09513590.2012.705377.

11. Parelkar SV, Mundada D, Sanghvi BV, et al. Should the ovary always be conserved in torsion? A tertiary care institute experience. J Pediatr Surg. 2014; 49: 465-8.

12. Bin Z, Yan G, Jingjing Li. Report of 62 cases of ovarian cyst pedicled to preserve ovarian laparoscopic surgery[J] . Chinese Journal of Minimally Invasive Surgery. 2014; 7(14): 600-602.

13. Spinelli C, Buti I, Pucci V, et al. Adnexal torsion in children and adolescents: new trends to conservative surgical approach - Our experience and review of literature. Gynecological Endocrinology. 2012; 29(1): 54-58, doi: 10.3109/09513590.2012.705377.

14. Kives S, Gascon S, Dobuc E, et al. Diagnosis and Management of Adnexal Torsion in Children, Adolescents, and Adults. J Obstet Gynaecol Can. 2017; 39(2), doi: 10.1016/j.jogc.2016.10.001. 